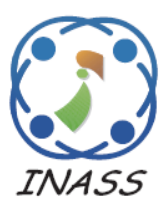

\title{
An approach based on Clustering AH and VND for Supply Chain Management
}

\author{
Fadoua Oudouar ${ }^{1 *}$ \\ El Miloud Zaoui ${ }^{2}$ \\ ${ }^{1}$ Mohammadia School of Engineers, Mohammed V University in Rabat, Morocco \\ ${ }^{2}$ National Superior School of Mines, Rabat, Morocco \\ * Corresponding author's Email: oudouar.fadoua@gmail.com
}

\begin{abstract}
In this work, we present a new approach to solve the Capacitated Location-Routing Problem (CLRP). The aim of this method is to determine the depot locations, to assign customers to facilities and to define routes for each depot to serve the associated clients. The proposed approach contains two phases, which are the constructive phase and the improvement phase. In the first phase, we select the depots to be opened, allocate the customers to open depots using a Hierarchical Ascendant (HA) method and we solve the vehicle routing problem for each depot using Sweep algorithm. In the second phase, we apply a Variable Neighborhood Descent (VND) with three structures in order to optimize the cost obtained by the first phase. Two sets of well-known instances from the literature are used to evaluate the performance of this method, and the numerical results obtained are compared with the experimental results of other methods. Results show that our method is competitive with respect to the best-known solutions (BKSs) and demonstrate its efficiency in comparison with other approaches.
\end{abstract}

Keywords: Capacitated location routing problem, Variable neighborhood search, Clustering, Hierarchical ascendant, Logistic problems.

\section{Introduction}

The Location Routing Problem (LRP) is a strategic problem of supply chain management. This problem includes two major logistic problems, namely, facility location and routing problems, which are both, belong to the class of NP-hard problem. Traditionally, these two types of problems have been determined and carried out at different levels. While the location is a strategic decision that must be made for a long time, the routing is an operational stage that can be solved more frequently in a short time. Since it was proved that the combination of location and routing decisions reduces the distribution cost and provides benefits for both operators and society. As a result, the LRP has become an interesting field of research [1].

Several variants of LRP have been studied in the literature such as LRP with simultaneous delivery and pickup [2], LRP with time windows. Due to large number of papers dealing with this problem, the authors of $[3,4]$ have written literature updates for a recent review of the LRP. More recently, the paper [5] presents an exact method for the location routing problem with time windows. In [6] the authors studied a robust Location Routing Problem for the design of electric logistics fleet networks was presented. The authors of [7] considered a new industrial hazardous waste location-routing problem with three objective functions and new mathematical formulation. Many studies on the LRP consider either capacitated depots or capacitated routes. Since most authors address the LRP with capacity constraint for both the depots and the routes [8].

In this paper, we consider the capacitated version of the LRP, in which capacity constraints on depots and vehicles must be respected, named Capacitated Location Routing Problem (CLRP). Several solution methods have been proposed for the solution of the CLRP. Exact methods were developed in [1] to solve the problem. However, the exact solution approaches display a good solution for small-sized instances. In light of the increasing complexity of CLRP, heuristic and meta-heuristic algorithms have been adopted to solve the CLRP. A new meta-heuristic algorithm 
based on two phases is proposed to solve the CLRP [9]. In addition, a Greedy Randomized Adaptive Search Procedure (GRASP) with a randomized version of the Clarke and Wright $(\mathrm{CW})$ algorithm proposed in [14]. The same authors developed a new meta-heuristic called the Lagrangean Relaxation Granular Tabu Search (LRGTS) [7]. Many researchers use clustering methods to assign customers to depots, these technics create the clusters using similarity between the data [10].

The main contribution of this work is to combine three algorithms to solve the capacitated location routing problem with homogeneous fleet. We propose a framework based on HA to find the locations of the facilities and the assignments of the customers, a sweep algorithm to determine the routes for each facility and VND with three neighborhood structures to improve the routes. The goal of the proposed approach is to solve a facility location problem and a vehicle routing problem simultaneously without violating capacity constraints for both depots and vehicles.

This paper is organized as follows. The section 2 describe the related work. The description of the Capacitated Location Routing Problem and mathematical formulation are defined in Section 3. Section 4 presents the details of the proposed approach. Section 5 reports computational results on the benchmark instances from the literature. Finally, the last section contains the conclusion and future direction.

\section{Related work}

The Capacitated Location Routing Problem is a specific problem of LRP in which the capacity constraints must be respected for both depots and vehicles. This problem combines two well-known NP-hard problems: the Capacitated Facility Location Problem and the Vehicle Routing Problem (VRP). Due to the complexity of the problem, several methods have been developed to solve the CLRP that can be classified into two principal types: exact and approximate algorithms [11].

A branch-and-cut algorithm based on an integer formulation for solving this problem is presented in [16]. Moreover, the authors in [5] proposed a branchand-cut-and-price algorithm to solve the problem. This method consists in formulating the CLRP in a set-partitioning formulation. These exact methods are able to solve only limited to medium size instances. For this reason, the research has focused on many heuristics and hybrid methods and try to approach the best solution. A greedy randomized adaptive search procedure (GRASP) method was proposed in [12].
According to authors in [13], a similar method has been hybridized with evolutionary local search for CLRP. This method is the first hybrid approach combining the variable neighborhood search and integer-linear programming technique for solving the CLRP. In addition, a VNS combined with an evolutionary local search (ELS) method (VNS $\times$ ELS) was developed by [14]. This approach based on seven different neighborhood structures to improve the solution. The authors in [15] present a Multiple Ant Colony Optimization (MACO) methods. This algorithm adopts a nested mechanism with three hierarchical ant colony structure. A simulated annealing based algorithm was introduced in [16] and tested on three sets of well-known benchmark instances for solving the CLRP. The study in [13] present a new heuristic called Granular Variable Tabu Neighborhood Search. This method combines a Granular Tabu Search and a Variable Neighborhood Search algorithm. A hybrid genetic algorithm with a local search in the mutation phase presented in [17], [18].

\section{Mathematical formulation for CLRP}

The capacitated Location Routing Problem can be defined on a complete, and undirected graph $G=$ $(V, E)$ in which $V=\{1, \ldots, m+n\}$ is a set of nodes and $E$ is the edge set. Vertices $i=1, \ldots, m$ correspond to the potential depot locations and vertices $j=m+1, \ldots, m+n$ correspond to the customers. Each potential depot $i$ has a capacity $w_{i}$ and an opening cost $o_{i}$. Each customer $j$ has nonnegative demand $d_{j}$ (which must be served by a depot). An unlimited set of homogeneous vehicles,

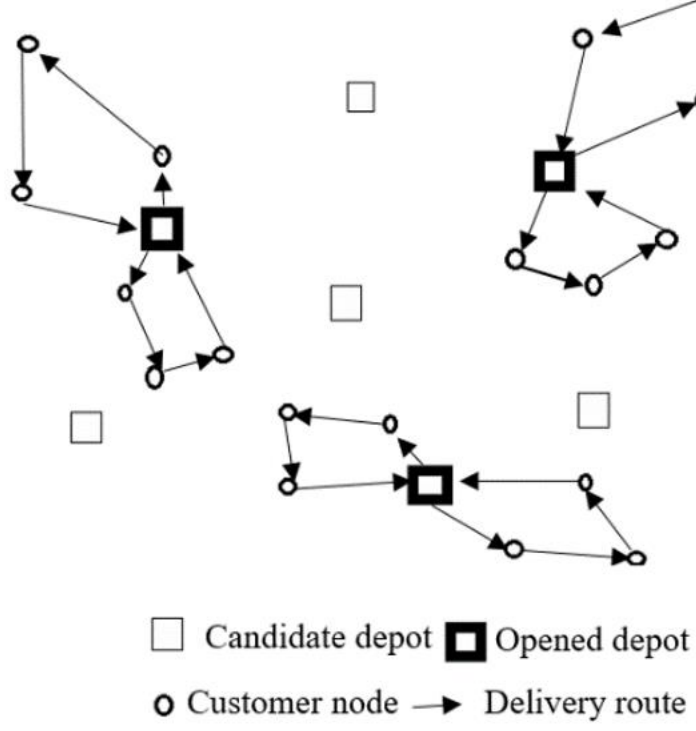

Figure. 1 Example of Location Routing Problem 
each with capacity $K$ and a nonnegative fixed cost $F$, is available at each depot. A traveling cost $c_{i j}$ is associated with each edge $(i, j) \in E$.

Fig. 1 represents an example of LRP with seven possible depot locations and 18 customers. Each customer is assigned to one open depot and served by an exactly one route.

A solution for the CLRP consists of finding the depots to be opened, and the routes to be constructed to serves the customers. Each route must begin and end at the same depot, the sum demand of each route must not exceed the vehicle capacity $Q$, and the global demand of customers assigned to a depot $i$ must respect its capacity $w_{i}$. The objective function of the CLRP is to minimize the sum of the costs of the open depots, of the fixed costs associated with the used vehicles, and of the costs of the edges traveled by the routes. Define the depot variables $y_{i}=1$ if depot $i$ is opened, and 0 otherwise. The edge variables $x_{i j}^{k}$ are equal to 1 if vehicle $k$ visits customer $j$ immediately after customer $i$. Finally, variables $f_{i j}=1$ is a customer $j$ is assigned to depot $i$. The mathematical model [19] is given by:

$$
\begin{array}{r}
\min z=\sum_{i \in I} O_{i} y_{i}+\sum_{i \in V} \sum_{j \in V} \sum_{k \in K} C_{i j k} x_{i j k}+ \\
\sum_{k \in K} \sum_{i \in I} \sum_{j \in J} F x_{i j k}
\end{array}
$$

Subject to

$$
\begin{gathered}
\sum_{k \in K} \sum_{i \in V} x_{i j k}=1 \quad \forall j \in J \\
\sum_{j \in J} \sum_{i \in V} d_{j} x_{i j k} \leq Q \quad \forall k \in K \\
\sum_{j \in V} x_{i j k}-\sum_{j \in V} x_{j i k}=0, \forall k \in K, \forall i \in V \\
\sum_{i \in I} \sum_{j \in J} x_{i j k} \leq 1 \quad \forall k \in K \\
\sum_{i \in S} \sum_{j \in S} x_{i j k} \leq|S|-1, \forall S \subseteq J, \forall k \in K \\
\sum_{u \in J} x_{i u k}+\sum_{u \in V \backslash\{j\}} x_{u j k} \leq 1 \\
+f_{i j} \forall i \in I, \quad \forall j \in J, \forall k \in K \\
\sum_{j \in J} d_{j} f_{i j} \leq W_{i} y_{i} \quad \forall i \in I \\
x_{i j k} \in\{0,1\} \quad \forall i \in V, \forall j \in V, \forall k \in K \\
y_{i} \in\{0,1\} \quad \forall i \in I \\
f_{i j} \in\{0,1\} \quad \forall i \in I, \forall j \in V
\end{gathered}
$$

In this formulation, the objective function (1) minimizes the total cost including depot opening and the traveling costs associated with the edges traversed by the routes.

Eq. (2) guarantee that each customer must be visited exactly once. Eqs. (3) and (8) are capacity constraints for both depots and vehicles. Eqs. (4) and (5) ensure the continuity of each route, and that each route starts and ends to the same depot. The elimination sub tour is ensured through inequalities Eq. (6). Eq. (7) provide that each cluster is assigned to exactly one depot. Finally, Eqs. (9) - (11) specify the binary variables used in the model.

\section{Proposed approach for solving CLRP}

Two strong points are the basis of our work. The first one is the use of new methods to generate feasible solutions to solve CLRP. While the second is to improve this one using a local search method. A solution of this problem consists of determining the depot to be opened, the customer to be assigned to each open depot, and the vehicle routes to be performed to serve all customers. For this reason, our method consists of two major phases: Construction phase and improvement phase. The Fig. 2 summarizes the steps of the proposed approach (named Hierarchical Ascendant and Variable Neighbourhood Descent, HA+VND).

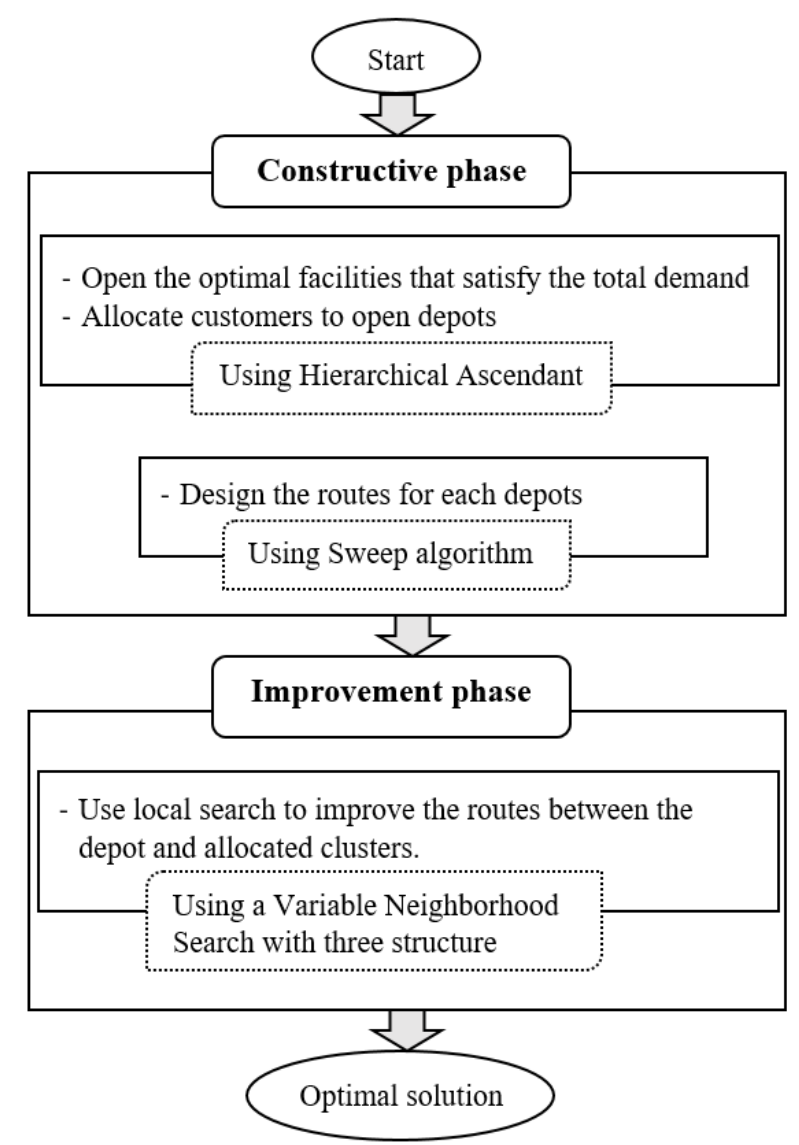

Figure. 2 Proposed framework HA+VND to solve CLRP 


\subsection{Construction phase}

In order to create an initial feasible solution, a two-step constructive method has been implemented. Location and allocation decisions are made in the first step while, routing decisions are determined in the second step. To find a solution for the facility location problem a Hierarchical Ascendant Clustering has been developed. Once the customers are affected to the depots, the problem becomes a Capacitated Vehicle Routing Problem, this problem is solved using a Sweep algorithm.

\section{Hierarchical ascendant clustering}

Hierarchical clustering algorithms is an important technique in data mining. This clustering algorithm is frequently used in several research areas such as data analytics, visualization, analyse Social networks [20].

In this method, customers are compared with one another based on their similarity. There are many criteria to compare customers based on their relationship. Clusters are built by joining small clusters of customers based on their similarity in terms of distance and demand. There are two types of hierarchical clustering approaches:

- Agglomerative approach: at the beginning, this method considers each customer as one cluster; after, the algorithm proceeds to merge the small clusters based on their similarities to construct the final clusters. This method is also called a bottomup approach.

- Divisive approach: Initially, this approach considers all data as a single cluster; after, each customer forms its proper cluster. The divisive approach is less widely used due to its complexity compared with the agglomerative approach. This method is also called a top-down approach.

In this work, our study based on Agglomerative approach. It requires an error measurement and a proximity function. The objective function is the sum of squared errors (SSE). The SSE for a set of customers $X=\left\{x_{1}, x_{2}, \ldots, x_{n}\right\}$ and for any given set $D$ of $m$ depots $\left\{D_{1}, D_{2}, \ldots, D_{m}\right\}$, is defined as:

$$
\operatorname{SSE}(k)=\sum_{i=1}^{m} \sum_{j=1}^{n_{i}} C\left(x_{i, j}, \overline{D_{l}}\right)^{2}
$$

where $c\left(x_{i, j}, \bar{D}_{l}\right)^{2}$ is the distance from the costomers $x_{i, j} \in D_{\mathrm{i}}$ to the location of depot $D_{i}$, and $n_{i}$ is the number of customers assigned to depotr $D_{i}$.

The hierarchical agglomerative algorithm is starting by the number of depot equal number of customers' $m=n$, and proceeds as follows:

Step 1. Calculate and store SSE(m).
Step 2. Build the distance matrix $d$ as

$C_{i j}=C\left(\bar{D}_{l}, \bar{D}_{J}\right), i=1, \ldots, m$ and $j=1, \ldots, m$

Step 3. Find the pair of depots with centroids $\overline{D_{p}}$ and $\overline{D_{q}}$ such that $d_{p q}$ is the minimum distance in $C$.

Step 4. Create a depot $D_{p q}=D_{p} \cup D_{q}$ where $p$, $\mathrm{q}$ are returned from $(p, q)=\min _{i j} D_{i j}$.

Step 5. Create a new set of depots, such that:

$$
\widehat{D}_{m-1}=\left(\left(\widehat{D}_{m} D_{p}\right) D_{p}\right) \cup D_{p q}
$$

where stands for the set subtraction operator.

Step 6. Update matrix $C$ including the centroid $\overline{\mathrm{D}}_{p q}$ for the new depot $\mathrm{D}_{p q}$.

The algorithm stops when the number of clusters (depots) equal $\mathrm{m}$. To calculate the number of depots, we use the customers' demand $\left(d_{j}\right)$ and the depot's capacity $w_{i}$ :

$$
N=\left\lceil\sum_{j=1}^{n} d_{j} / w_{i}\right\rceil
$$

\section{Sweep algorithm}

A large number of different heuristic algorithms have been widely applied in many routing problems. One of the simplest is Sweep Algorithm. This method was firstly introduced in [21] for capacitated vehicle routing problem (CVRP). The Sweep Algorithm is one of the known two phases constructive heuristic. At the first stage, all customers are clustered based on their $x-y$ coordinates. Then, the locations of each customer are according to the polar- coordinate angle. The depot is located at the center of the twodimensional plane.

This polar coordinate angle calculated by the following equation:

$$
\theta(i)=\arctan \left|\left(\frac{y_{i}-y_{0}}{x_{i}-x_{0}}\right)\right|
$$

Where 0 represent the depot.

In the second phase, the customers are clustered according to the polar angle in a clockwise manner. The maximum number of customers assigned to the current cluster depends on the capacity of the vehicle. Next, a route is generated by linking (connecting) neighbours in angular proximity in every cluster.

The formula for clustering customers is as follows:

$$
d_{i}+\sum_{j}^{i-1} d_{j} \leq Q \quad i \neq j
$$

The sweep procedure is described in the first algorithm. 


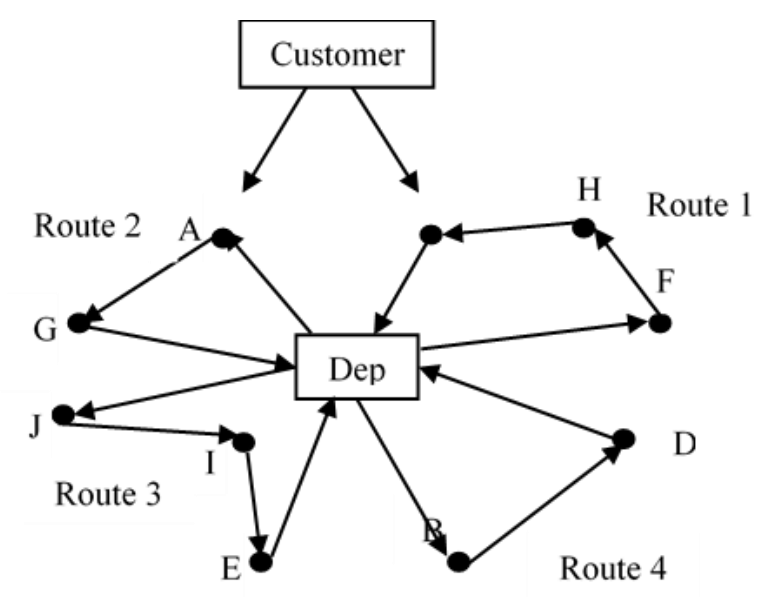

Figure. 3 Example of sweep algorithm

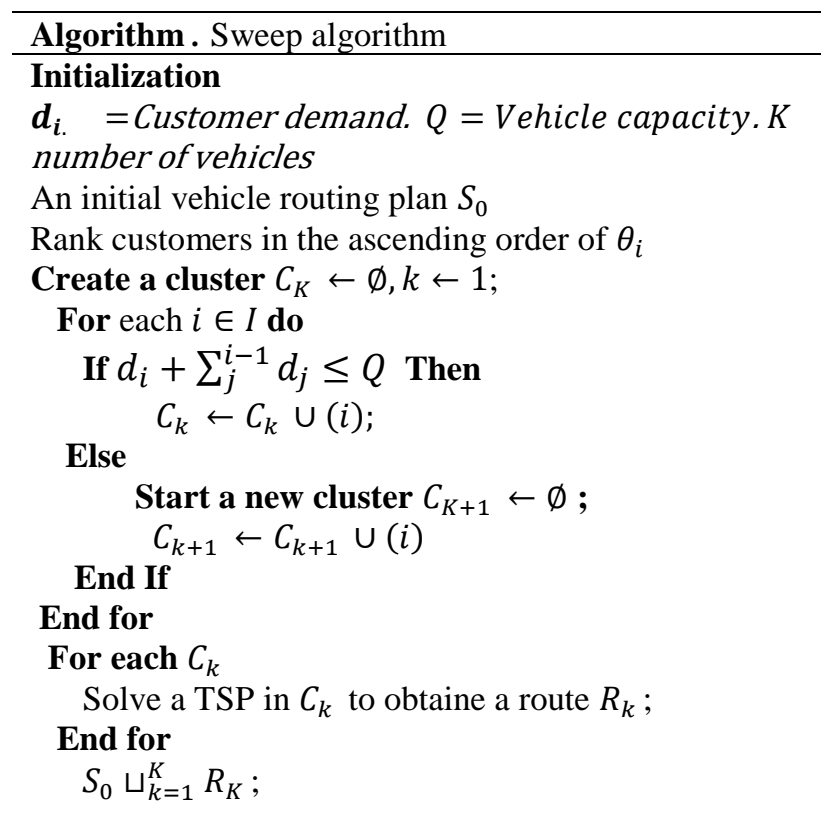

To understand the Sweep method clearly, an example with one depot and ten customers with different demands is considered, which is illustrated in Fig. 3. This figure presents the cluster phase starting from $0^{\circ}$ : the first customer is one which has smallest angle, then select the second closest, the third, and so forth until it satisfies the capacity constraint.

\subsection{Improvement phase}

In this phase, our approach tries to improve the initial solution obtained by the construction phase using a variant of a Variable neighborhood search VNS. The objective of the improvement phase is to optimize the routes. For this reason, three neignborhood structures are used to find a good solution and try to locally improve it.

\section{Variable neighborhood search}

Variable Neighborhood Search (VNS) is a local search based metaheuristic proposed by [22]. The VNS approach is shown to be promising for solving difficult and global optimization problems. The main idea of this algorithm is based on a systematic change of neighborhood within a local search method [23]. It explores a set of predefined neighborhood to provide a better solution.

VNS is a stochastic algorithm in which, a set of neighborhood structures $N_{k}, k \in\left\{1, \ldots, k_{\max }\right\}$ are explored during the search. The VNS start from an initial solution, and a set $N_{k}(x)$ of solutions of the Kth neighborhood of $x$. At each iteration, a solution $x^{\prime}$ is then randomly selected and computed with respect to the $K^{\text {th }}$ neighborhood, $N_{k}(x)$. Then, a local search procedure is applied to the solution $x^{\prime}$ to generate a second solution $x^{\prime \prime}$. If the solution $x^{\prime \prime}$ is better than $x$, the solution $x^{\prime \prime}$ becomes the new current solution and the search starts from the update solution. Otherwise, the same operations are repeated with the next neighborhood $N_{k+1}$. The current solution will be a local optimum with respect to the neighborhood structure used. There are two local operators: The single-route consists of exchanging the position of a customer in a single route. The multi-route is used to remove a customer from its current position and affecting it into a new position in another route.

More recently, VNS has proved its effectiveness to solve VRPs. We find several works such as [24], they used this method for solving a VRP with deliveries and selective pick-ups. The same for [25], these authors solved a capacitated vehicle routing problem using this approach. [26] proposed a simpler Variable Neighborhood Search heuristic for Vehicle Routing Problem with Multiple Time Windows. Also, many authors have been combining the VNS with other heuristics to solve several NP-hard routing problems.

In this section, we describe the variant of VNS named Variable Neighborhood Descend (VND). The main idea of VND is to explore a set of predefined operators to get different local optima to provide a better solution. This method starts by an initial solution $x$ obtained by the construction phase, as the starting point of search. Then, we define a set of neighborhood structures $N_{k}\left(k=1, \ldots, k_{\max }\right)$. Let $N_{1}$ be the first neighborhood operators to be used. At each step of the search procedure, if an improvement of the current solution $x$ is found, the search start with the same neighborhood structures $N_{1}(x)$. On the other hand, if it is not possible to find an 
improvement of the solution $x$ in $N_{1}(x)$, the operators is changed from $N_{k}$ to $N_{k+1}$.

The proposed algorithm VND incorporates three neighborhood structures to improve route assignment to each depot. The neighborhood operators used in the VND procedure are shift move, swap move and 2-opt move.

Shift move: This first neighborhood consists in moving a customer present in a route, either from its current position to another position in the within the same route or in another route. These neighborhoods are described below.

- Swap $(1,1)$ : the basic idea is to permute a customer $a$ from a route $r_{i}$ and a customer $m$ from a route $r_{j}$.

- Shift $(1,0)$ : The basic idea is to change the location of a customer $a$ from a route $r_{i}$ to a route $r_{j}$.

- two-Opt: This operator enables the delete of two arcs in a route and two others of the same route are added.

\section{Computational results and discussion}

The proposed method (HA+VND) has been implemented in $\mathrm{C}++$, and the computational results have been performed on a Computer I3, 8G RAM. The algorithm has been evaluated by considering two well-known benchmark sets in the literature. The complete set of instances considers capacity constraints. The first set was proposed by Tuzun and Burke [9] and contains 36 benchmark instances. These instances consider 10 and 20 potential depots as well as 100, 150, and 200 customers. The values for the vehicle capacity is set to 150 . The second data set is gathered by Barreto [27] and considers 13 benchmark instances obtained by adding new depots with capacities. The number of customers varies from 21 to 150 , and the number of potential facilities from 5 to 10. In all sets of benchmark, the depots and customers are represented by points in the plan. As a result, the traveling cost of an edge corresponds to the Euclidean distance, calculated as a real number.

Our results have been compared with two heuristics proposed in the literature for the solution of CLRP. These algorithms are the Cooperative Approximation Heuristic Algorithm CAHA [28] and Hybrid GA+ [29]. The experiments consider two types of comparison because the first method was executed only once, while the second was executed for ten runs. The description of this method is given in Table 1.
Table 1. Information of two methods proposed in the literature of the CLRP

\begin{tabular}{|c|l|c|c|}
\hline Method & \multicolumn{1}{|c|}{ Authors } & $\begin{array}{c}\text { N. } \\
\text { Runs }\end{array}$ & $\begin{array}{c}\text { T. } \\
\text { Sol }\end{array}$ \\
\hline CAHA & $\begin{array}{l}\text { Q. Zhao, W. Wang, and R. } \\
\text { D. Souza }\end{array}$ & 1 & best \\
\hline $\begin{array}{c}\text { Hybrid } \\
\text { GA+ }\end{array}$ & $\begin{array}{l}\text { R. B. Lopes, C. Ferreira, } \\
\text { and B. S. Santos }\end{array}$ & 10 & best \\
\hline
\end{tabular}

- N. Runs: Number of runs

- T. Sol: Type of solutions

In Table 2 and Table 3, the results of the proposed approach in the capacitated location routing problem for the two data set of instances are presented. They are also compared with the two methods in terms of the best cost and the relative percentage gap, calculated as: $100 \times\left(\frac{\operatorname{Cost}-B K S}{B K S}\right)$, where Cost denotes the cost of the solution found by each method and $B K S$ the cost of the best known-solution. In each row of Tables 2-3, the following can be seen: the name of instances, with the set of $\mathrm{n}$ customers and $\mathrm{m}$ candidate depots $(n \times m)$. The value of the best-known solution (BKS). The last row presents the detail information of the best cost (Cost) and percentage gap of each method.

The results of the first dataset are given in Table 2. It can be seen that the proposed approach in twenty-five out of thirty-six instances has reached the best-known solutions. For the rest instances, the quality of the solution is between 0.07 and 3.63. The average quality of the 36 instances is equal to 0.37 . Our method gives better results from the two other algorithm. It is, also, to note that the effectiveness of this approach improves, with respect to that of the other heuristics, for the largest instances (150 and 200 customers).

In Table 3, the results in the second dataset are presented. It can be seen that the proposed method in three out of thirteen instances has reached the bestknown solutions. For the other instances, the quality of the solution is between 0.13 to 0.55 . In addition, the results show that the average deviations is small than 1 in all instances. The average quality of the 19 instances is equal to 0.25 .

In addition, a comparison between the HA+VND, the CAHA and the Hybrid GA+ has also been summarized in Fig. 4. This three methods have been tested in 49 instances, they have average quality equal to 0,31 for the proposed approach, 1.12 for the hybrid GA+ and 2.17 for the CAHA. The reported results indicate that our method is competitive compared to the existing solution approaches for the CLRP.

In summary, the proposed method is tested in 49 CLRP benchmark instances. The algorithm found the 
Table 2. Computational results for the instances of tuzun and burke

\section{BKS \\ CAHA \\ Hybrid GA+ \\ HA+VND}

\begin{tabular}{|c|c|c|c|c|c|c|c|c|c|}
\hline & $\mathbf{n}$ & $\mathbf{m}$ & & Cost & Gap/BKS & Cost & Gap/ BKS & Cost & Gap/BKS \\
\hline 111112 & 100 & 10 & 1468,4 & 1482,58 & 0,97 & 1469,54 & 0,08 & 1473,16 & 0,32 \\
\hline 111122 & 100 & 20 & 1449,2 & 1514,97 & 4,54 & 1449,2 & 0,00 & 1492,24 & 2,97 \\
\hline 111212 & 100 & 10 & 1395,8 & 1403,52 & 0,55 & 1395,8 & 0,00 & 1401,54 & 0,41 \\
\hline 111222 & 100 & 20 & 1424,7 & 1480,12 & 3,89 & 1432,29 & 0,53 & 1424,7 & 0,00 \\
\hline 112112 & 100 & 10 & 1167,53 & 1170,84 & 0,28 & 1167,53 & 0,00 & 1170,26 & 0,23 \\
\hline 112122 & 100 & 20 & 1095,26 & 1113,72 & 1,69 & 1102,38 & 0,65 & 1095,26 & 0,00 \\
\hline 112212 & 100 & 10 & 787,7 & 804,19 & 2,09 & 791,91 & 0,53 & 787,7 & 0,00 \\
\hline 112222 & 100 & 20 & 706,12 & 735,27 & 4,13 & 730,27 & 3,42 & 706,12 & 0,00 \\
\hline 113112 & 100 & 10 & 1216,89 & 1266,12 & 4,05 & 1238,49 & 1,78 & 1216,89 & 0,00 \\
\hline 113122 & 100 & 20 & 1246,06 & 1259,96 & 1,12 & 1246,06 & 0,00 & 1252,48 & 0,52 \\
\hline 113212 & 100 & 10 & 901,52 & 903,82 & 0,26 & 903,5 & 0,22 & 901,52 & 0,00 \\
\hline 113222 & 100 & 20 & 1019,39 & 1034,72 & 1,50 & 1019,39 & 0,00 & 1022,67 & 0,32 \\
\hline 131112 & 200 & 10 & 1866,75 & 1891,97 & 1,35 & 1921,3 & 2,92 & 1934,58 & 3,63 \\
\hline 131122 & 200 & 20 & 1830,75 & 1848,79 & 0,99 & 1830,75 & 0,00 & 1846,7 & 0,87 \\
\hline 131212 & 200 & 10 & 1891,37 & 1989 & 5,16 & 1981,26 & 4,75 & 1953,58 & 3,29 \\
\hline 131222 & 200 & 20 & 1804,43 & 1815,12 & 0,59 & 1813,72 & 0,51 & 1804,43 & 0,00 \\
\hline 132112 & 200 & 10 & 1438,62 & 1465,93 & 1,90 & 1457,01 & 1,28 & 1438,62 & 0,00 \\
\hline 132122 & 200 & 20 & 1444,25 & 1453,72 & 0,66 & 1446,46 & 0,15 & 1445,26 & 0,07 \\
\hline 132212 & 200 & 10 & 1203,59 & 1208,6 & 0,42 & 1211,83 & 0,68 & 1203,59 & 0,00 \\
\hline 132222 & 200 & 20 & $\mathbf{9 2 7 , 8 2}$ & 932,19 & 0,47 & 934,37 & 0,71 & $\mathbf{9 2 7 , 8 2}$ & 0,00 \\
\hline 133112 & 200 & 10 & 1690,92 & 1699,92 & 0,53 & 1708,63 & 1,05 & 1690,92 & 0,00 \\
\hline 133122 & 200 & 20 & 1400,01 & 1418,66 & 1,33 & 1400,01 & 0,00 & 1411,56 & 0,82 \\
\hline 133212 & 200 & 10 & 1191,93 & 1199,51 & 0,64 & 1213,93 & 1,85 & 1191,93 & 0,00 \\
\hline 133222 & 200 & 20 & 1148,59 & 1150,71 & 0,18 & 1157,11 & 0,74 & 1148,59 & 0,00 \\
\hline 121112 & 150 & 10 & 2265,21 & 2281,78 & 0,73 & 2270,18 & 0,22 & 2265,21 & 0,00 \\
\hline 121122 & 150 & 20 & 2151,67 & 2269,42 & 5,47 & 2177,32 & 1,19 & 2151,67 & 0,00 \\
\hline 121212 & 150 & 10 & 2228,63 & 2269,09 & 1,82 & 2230,37 & 0,08 & 2228,63 & 0,00 \\
\hline 121222 & 150 & 20 & 2235,67 & 2257,43 & 0,97 & 2250,38 & 0,66 & 2235,67 & 0,00 \\
\hline 122112 & 150 & 10 & 2070,61 & 2103,87 & 1,61 & 2101,75 & 1,50 & 2070,61 & 0,00 \\
\hline 122122 & 150 & 20 & 1692,01 & 1743,08 & 3,02 & 1712,6 & 1,22 & 1692,01 & 0,00 \\
\hline 122212 & 150 & 10 & 1452,58 & 1476,4 & 1,64 & 1466,64 & 0,97 & 1452,58 & 0,00 \\
\hline 122222 & 150 & 20 & 1082,25 & 1101,72 & 1,80 & 1087,91 & 0,52 & 1082,25 & 0,00 \\
\hline 123112 & 150 & 10 & 1924,46 & 1983,18 & 3,05 & 1976,97 & 2,73 & 1924,46 & 0,00 \\
\hline 123122 & 150 & 20 & 1934,44 & 1983,21 & 2,52 & 1951,47 & 0,88 & 1934,44 & 0,00 \\
\hline 123212 & 150 & 10 & 1759,92 & 1772,12 & 0,69 & 1772,91 & 0,74 & 1759,92 & 0,00 \\
\hline 123222 & 150 & 20 & 1389,51 & 1399,89 & 0,75 & 1399,82 & 0,74 & 1389,51 & 0,00 \\
\hline Average & & & & 1524,59 & 1,76 & 1511,7 & 0,93 & 1503,59 & $\mathbf{0 , 3 7}$ \\
\hline
\end{tabular}


Table 3. Computational results for the instances of barreto

\begin{tabular}{|c|c|c|c|c|c|c|c|c|c|}
\hline \multirow[b]{2}{*}{ Instances } & \multirow[b]{2}{*}{$\mathbf{n}$} & \multirow[b]{2}{*}{$\mathbf{m}$} & \multirow[b]{2}{*}{ BKS } & \multicolumn{2}{|c|}{ САНА } & \multicolumn{2}{|c|}{ Hybrid GA+ } & \multicolumn{2}{|c|}{ HA+VND } \\
\hline & & & & Cost & $\begin{array}{l}\text { Gap/ } \\
\text { BKS }\end{array}$ & Cost & Gap/BKS & Cost & Gap/BKS \\
\hline christo 50 & 50 & 5 & 565,6 & 582,9 & 3,06 & 565,6 & 0,00 & 566,41 & 0,14 \\
\hline christo 75 & 75 & 10 & 838,73 & 864,8 & 3,11 & 844,4 & 0,68 & 838,73 & 0 \\
\hline christo100 & 100 & 10 & 833,6 & 845,9 & 1,48 & 833,6 & 0,00 & 837,94 & 0,52 \\
\hline Daskin95-88x8 & 88 & 8 & 353,57 & 382,6 & 8,21 & 355,8 & 0,63 & 353,57 & $\mathbf{0}$ \\
\hline Daskin95-150x10 & 150 & 10 & 43919,9 & 44011,7 & 0,21 & 43919,9 & 0,00 & 44160,34 & 0,55 \\
\hline Gaskell67-21x5 & 21 & 5 & 424,9 & 462,9 & 8,94 & 424,9 & 0,00 & 426,23 & 0,31 \\
\hline Gaskell67-22x5 & 22 & 5 & 585,1 & 590,1 & 0,85 & 585,1 & 0,00 & 587,86 & 0,47 \\
\hline Gaskell67-29x5 & 29 & 5 & 506,15 & 518,5 & 2,44 & 512,1 & 1,18 & 506,15 & $\mathbf{0}$ \\
\hline Gaskell67-32x5 & 32 & 5 & 562,2 & 571,7 & 1,69 & 562,2 & 0,00 & 563,98 & 0,32 \\
\hline Gaskell67-32x5 & 32 & 5 & 504,3 & 504,3 & 0,00 & 504,3 & 0,00 & 506,59 & 0,45 \\
\hline Gaskell67-36x5 & 36 & 5 & 460,4 & 467,7 & 1,59 & 460,4 & 0,00 & 461,24 & 0,18 \\
\hline Min92-27x5 & 27 & 5 & 3062 & 3067,7 & 0,19 & 3062 & 0,00 & 3067,01 & 0,16 \\
\hline Min92-134x8 & 134 & 8 & 5709 & 5809 & 1,75 & 5709 & 0,00 & 5716,43 & 0,13 \\
\hline Average & & & 4490,15 & 4504,16 & 2,58 & 4487,64 & 0,19 & 4507,11 & 0,25 \\
\hline
\end{tabular}

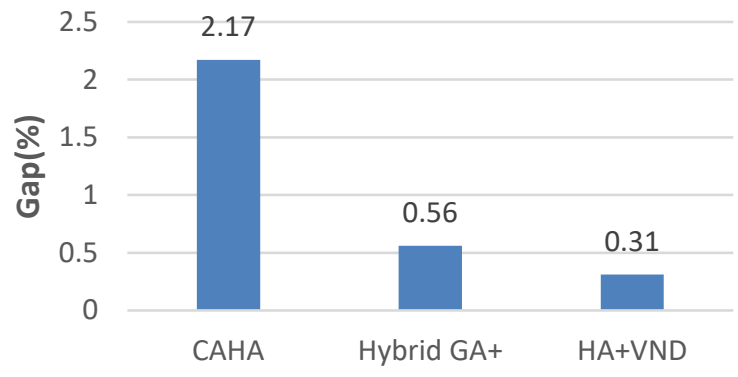

Algorithms

Figure. 4 Comparison average gap

new best known solution in 28 instances in a single run. The comparative results of the performance of the proposed approach with other algorithms in the literature indicate that our method can get better solution for the small, median and large instances.

\section{Conclusion and future works}

This paper has presented an efficient approach for solving the CLRP. The proposed method is presented in two phases; in the first phase, the facility location is performed and the customers are clustered using Hierarchical Ascendant heuristic (HA). While the routing is performed using Sweep heuristic. In the second phase, a variable neighborhood Decsent (VND) with three neighborhood operators is proposed to improve the best routes between the depot and allocated clusters.
The proposed method is tested on two sets of instances from the literature. The computational results show that our approach is able to provide competitive results when compared to those of the best performing methods, in term of solution quality. Out of 49 CLRP benchmark instances, we have 28 new best-known solutions. We obtained feasible solutions with the average percentage gap of 0,37 for the first benchmark, and 0,25 for the second. The main advantage of our method is relatively easy to implement and understand. Moreover, it may be applied to other problems that contain multiple phases.

As recommendation for future research lines on the CLRP can be mentioned to using other inventory policies such us using heterogeneous fleet, developing multi-objective function. It could be also interesting to other objective functions such as maximization of customer satisfaction and minimization of the risk.

\section{Conflicts of Interest}

The authors declare no conflict of interest.

\section{Author Contributions}

In this work, a two-phase hybrid heuristic is proposed for solving the CLRP. This problem integrates two NP-hard problems: the facility location and capacitated vehicle routing problems. 
Our approach consists of two steps, the first one is a constructive heuristic for generating an initial solution, while the second step is a local search to improve the solution found in the first step. The proposed method in the constructive step is based on the cluster-first and route second approach, where the facility location is performed using Hierarchical Ascendant heuristic, while the routing is performed using Sweep heuristic to solve Capacitated Vehicle Routing Problem in each depot. A variable neighborhood search based on three neighborhood operators is proposed to improve the solution. The proposed method has been implemented in $\mathrm{C}++$, and the computational results have been performed on a Computer I3, 8G RAM. The algorithm has been evaluated by considering two well-known benchmark sets in the literature (Tuzun\&Burke instances and Barreto instances). The computational results have shown that the proposed approach is a promising algorithm to solve the Capacitated Location Routing Problem. It improves several previously best-known solutions.

Both authors F. Oudouar and E.M Zaoui have participated and collaborated to realize this work.

\section{References}

[1] V. C. Hemmelmayr, J.-F. Cordeau, and T. G. Crainic, "An adaptive large neighborhood search heuristic for Two-Echelon Vehicle Routing Problems arising in city logistics", Comput. Oper. Res., Vol. 39, No. 12, pp. 32153228, Dec. 2012, doi: 10.1016/j.cor.2012.04.007.

[2] Y. Yu, S. Wang, J. Wang, and M. Huang, "A branch-and-price algorithm for the heterogeneous fleet green vehicle routing problem with time windows", Transp. Res. Part B Methodol., Vol. 122, pp. 511-527, 2019, doi: 10.1016/j.trb.2019.03.009.

[3] C. Prodhon and C. Prins, "A survey of recent research on location-routing problems", Eur. J. Oper. Res., Vol. 238, No. 1, pp. 1-17, Oct. 2014, doi: 10.1016/j.ejor.2014.01.005.

[4] M. Drexl and M. Schneider, "A survey of variants and extensions of the location-routing problem", Eur. J. Oper. Res., Vol. 241, No. 2, pp. 283-308, 2015, doi: 10.1016/j.ejor.2014.08.030.

[5] S. Ponboon, A. G. Qureshi, and E. Taniguchi, "Branch-and-price algorithm for the locationrouting problem with time windows", Transp. Res. Part E Logist. Transp. Rev., Vol. 86, pp. 119, 2016, doi: 10.1016/j.tre.2015.12.003.

[6] M. Schiffer and G. Walther, "Strategic planning of electric logistics fleet networks: A robust location-routing approach", Omega, Vol. 80, pp. 31-42, 2018, doi: 10.1016/j.omega.2017.09.003.

[7] M. Rabbani, R. Heidari, H. Farrokhi-Asl, and N. Rahimi, "Using metaheuristic algorithms to solve a multi-objective industrial hazardous waste location-routing problem considering incompatible waste types", J. Clean. Prod., Vol. 170, pp. 227-241, 2018, doi: 10.1016/j.jclepro.2017.09.029.

[8] F. Oudouar, M. Lazaar, and Z. E. Miloud, "A novel approach based on heuristics and a neural network to solve a capacitated location routing problem", Simul. Model. Pract. Theory, Vol. $100, \quad$ p. 102064, 2020, doi: 10.1016/j.simpat.2019.102064.

[9] D. Tuzun and L. I. Burke, "A two-phase tabu search approach to the location routing problem", Eur. J. Oper. Res., Vol. 116, No. 1, pp. 87-99, 1999, doi: 10.1016/S0377-2217(98)00107-6.

[10] N. Rijati, D. Purwitasari, S. Sumpeno, and M. Purnomo, "A Decision Making and Clustering Method Integration based on the Theory of Planned Behavior for Student Entrepreneurial Potential Mapping in Indonesia", International Journal of Intelligent Engineering and Systems, Vol. 13, No. 4, pp. 129-144, 2020, doi: 10.22266/ijies2020.0831.12.

[11] F. Oudouar, A. El Fallahi, and E. M. Zaoui, "An improved heuristic based on clustering and genetic algorithm for solving the multi-depot vehicle routing problem", International Journal of Recent Technology and Engineering, pp. 6535-6540, 2019.

[12] C. Contardo, J.-F. Cordeau, and B. Gendron, "A GRASP + ILP-based metaheuristic for the capacitated location-routing problem," $J$. Heuristics, Vol. 20, No. 1, pp. 1-38, 2014, doi: 10.1007/s10732-013-9230-1.

[13] J. W. Escobar, R. Linfati, P. Toth, and M. G. Baldoquin, "A hybrid Granular Tabu Search algorithm for the Multi-Depot Vehicle Routing Problem", J. Heuristics, Vol. 20, No. 5, pp. 483509, 2014, doi: 10.1007/s10732-014-9247-0.

[14] E. Pekel and S. Soner Kara, "Solving fuzzy capacitated location routing problem using hybrid variable neighborhood search and evolutionary local search", Appl. Soft Comput., Vol. 83, p. 105665, 2019, doi: 10.1016/j.asoc.2019.105665.

[15] C.-J. Ting and C.-H. Chen, "A multiple ant colony optimization algorithm for the capacitated location routing problem", Int. J. Prod. Econ., Vol. 141, No. 1, pp. 34-44, 2013, doi: 10.1016/j.ijpe.2012.06.011. 
[16] V. F. Yu, S.-W. Lin, W. Lee, and C.-J. Ting, “A simulated annealing heuristic for the capacitated location routing problem", Comput. Ind. Eng., Vol. 58, No. 2, pp. 288-299, 2010, doi: 10.1016/j.cie.2009.10.007.

[17] W. Ho, G. T. S. Ho, P. Ji, and H. C. W. Lau, “A hybrid genetic algorithm for the multi-depot vehicle routing problem", Eng. Appl. Artif. Intell., Vol. 21, No. 4, pp. 548-557, 2008, doi: 10.1016/j.engappai.2007.06.001.

[18] Y. Barradi, N. Khaldi, K. Zazi, and M. Zazi, "A Novel Genetic Approach Applied for Power Loss Reduction and Improved Bus Voltage Profile in Distribution Network System", International Journal of Intelligent Engineering and Systems, Vol. 12, No. 6, pp. 91-99, 2019, doi: 10.22266/ijies2019.1231.09.

[19] C. Prins, C. Prodhon, and R. W. Calvo, "Solving the capacitated location-routing problem by a GRASP complemented by a learning process and a path relinking", 4OR, Vol. 4, No. 3, pp. 221-238, 2006, doi: 10.1007/s10288-006-00019.

[20] S. Chakraborty, D. Paul, and S. Das, "Hierarchical clustering with optimal transport," Stat. Probab. Lett., Vol. 163, p. 108781, 2020, doi: $10.1016 / j . s p l .2020 .108781$.

[21] B. E. Gillett and L. R. Miller, "A Heuristic Algorithm for the Vehicle-Dispatch Problem", Oper. Res., Vol. 22, No. 2, pp. 340-349, 1974, doi: 10.1287/opre.22.2.340.

[22] N. Mladenović and P. Hansen, "Variable Neighborhood Search", Comput Oper Res, Vol. 24, No. 11, pp. 1097-1100, 1997, doi: 10.1016/S0305-0548(97)00031-2.

[23] S. Nunes Bezerra, M. J. F. Souza, S. R. de Souza, and V. Nazário Coelho, "A VNS-Based Algorithm with Adaptive Local Search for Solving the Multi-Depot Vehicle Routing Problem", in Variable Neighborhood Search, Cham, pp. 167-181, 2019, doi: 10.1007/978-3030-15843-9_14.

[24] I. M. Coelho, P. L. A. Munhoz, L. S. Ochi, M. J. F. Souza, C. Bentes, and R. Farias, "An integrated CPU-GPU heuristic inspired on variable neighbourhood search for the single vehicle routing problem with deliveries and selective pickups", Int. J. Prod. Res., Vol. 54, No. 4, pp. 945-962, 2016, doi: 10.1080/00207543.2015.1035811.

[25] M. Amous, S. Toumi, B. Jarboui, and M. Eddaly, "A variable neighborhood search algorithm for the capacitated vehicle routing problem", Electron. Notes Discrete Math., Vol. 58, pp.
231-238,

2017 ,

doi:

10.1016/j.endm.2017.03.030.

[26] K. M. Ferreira and T. A. de Queiroz, "Two effective simulated annealing algorithms for the Location-Routing Problem", Appl. Soft Comput., Vol. 70, pp. 389-422, 2018, doi: 10.1016/j.asoc.2018.05.024.

[27] S. dos S. Barreto, Modelização de Problemas de localização-distribuição, Vol. Unpublished doctoral dissertation, University of Aveiro. Portugal, 2004.

[28] Q. Zhao, W. Wang, and R. D. Souza, "A heterogeneous fleet two-echelon capacitated location-routing model for joint delivery arising in city logistics", Int. J. Prod. Res., Vol. 56, No. 15, pp. 5062-5080, 2018, doi: 10.1080/00207543.2017.1401235.

[29] R. B. Lopes, C. Ferreira, and B. S. Santos, "A simple and effective evolutionary algorithm for the capacitated location-routing problem", Comput. Oper. Res., Vol. 70, pp. 155-162, 2016, doi: 10.1016/j.cor.2016.01.006. 